\title{
Cooperative Behavior based on a Subjective Map with Shared Information in a Dynamic Environment
}

\author{
Noriaki Mitsunaga*†, Taku Izumi*, and Minoru Asada*† \\ \{mitchy,izumi\}@er.ams.eng.osaka-u.ac.jp asada@ams.eng.osaka-u.ac.jp
}

\begin{abstract}
This paper proposes a subjective map representation that enables a robot in a multiagent system to make decisions in a dynamic, hostile environment. A typical situation can be found in the Sony four-legged robot league of the RoboCup competition [1]. The subjective map is a map of the environment that each agent maintains regardless of the objective consistency of the representation among the agents. Owing to the map's subjectivity, it is not affected by incorrect information aquired by other agents. The method is compared with conventional methods with or without information sharing.
\end{abstract}

Keywords: subjective map, multiagent, cooperative behavior, communication, dynamic environment

\section{INTRODUCTION}

In a multi-robot system, communication is expected to help robots acquire knowledge about the environment. Robots require a common reference coordinate system to exchange information about the environment. A world coordinate system is such an example. To convert the observation results to the world coordinates, each robot has to localize itself. However, localization errors often become too large to ignore. Kurazume et al. [2] have proposed a cooperative positioning system for a group of robots to cope with the errors from dead-reckoning. A robot can track its position by using dead-reckoning even in unknown environment, but accumulated errors are not negligible. Their idea is to always keep some robots stand still and other moving robots use them as landmarks. Then, the robots can avoid to use dead-reckoning. The drawback is that robots have to move in a group and be equipped with some device to accurately measure relative position between robots.

Means of localizing robots and acquiring their spatial configuration through their sharing of information have been proposed [3, 4, 5]. Kato et al. [4] and Nakamura et al. [5] use geometric constraints between several robots. Robots simultaneously observe each other, search their spatial configurations, which satisfy the constraints of the triangle formed by their positions, and share the map of the environment. For the global localization, one robot's position is used as the base, then localization errors do not influence information exchange, but depend on the self-localization of the base robot. To observe 
several robots at a glance, they used omnidirectional cameras rather than normal cameras with limited viewing angles. However, there will be many situations in which robots using conventional cameras will not be able to observe other robots. Then it becomes difficult to use such methods.

Although beliefs and a probabilistic representation of self-location is commonly used to cope with self-localization error, it is difficult to obtain an accurate model for merging the maps of several robots and maintaining the merged map. A simple weighted average of the information from each robots may work when the errors are small and modeled. Fox et al. [6] proposed a cooperative Markov localization method for a multi-robot system. Each robot localizes itself and merges other robots' observation based on global positioning in the world coordinate system. They do not assume the simultaneous observation of each other as in $[3,4,5]$ but assume the observation error can be modeled in advance and communication delay is negligible. Then, when one of the robots has a large error which are not modeled in its self-localization, it will affect the map used by other robots with either method. It is difficult to design the weights or to model in order to prevent this because there are always errors that the designer cannot anticipate, and in many situations the robot cannot detect modeling errors.

Scott et al. [7] proposed resetting of the Monte Carlo localization. A robot discards its past observations and resets its self-localization when it detects large inconsistency in its self localization with its sensor measurements. They showed experimental results in the RoboCup environment. Their method improves the reliability of the self-localization. However, there will be delay before the resetting is taken and errors affect the other robots which use a shared map.

We propose an approach that uses subjective maps instead of a shared map. A subjective map is for a robot in a multiagent system to make decisions in a dynamic, hostile environment. We assume that relative positions from a robot itself are more important than absolute locations in an environment and a robot can measure more precisely relative positions than absolute positions of objects in the environment. The subjective map is maintained by each robot regardless of the objective consistency of representations among the other robots. Owing to its subjectivity, incorrect information aquired by other agents does not affect the method. For example, accidents or other non-negligible changes in the environment do not affect the map. A potential field is defined on the subjective map in terms of subtasks, such as approaching or shooting the ball, and the field is dynamically updated so that the robot can decide what to do next. The method is compared with methods that involve sharing or not sharing and then future issues are discussed.

\section{GENERATION OF THE SUBJECTIVE MAP}

In a robot soccer task, there are teammate and opponent robots, a ball, and some landmarks. A robot can discriminate teammates and opponents but cannot identify them. Each robot does the following:

1. localize itself,

2. estimate the location of the ball, the teammates, and the opponents in the global coordinate 
system, if it observes any of them,

3. broadcast the results of its localization and estimated locations of the above to its other teammates,

4. receive messages from teammates,

5. construct its subjective map,

(a) if both the robot and its teammate observe the ball, it shifts the locations sent by the teammate based on the ball location and adds it to its subjective map,

(b) in other cases, the robot just adds objects, including its teammate, which it does not observe currently, to its subjective map, and

6. calculate the potential field and determine its action.

Here, we show an example of a subjective map. We assume that there are two robots (robot A and robot B) and a ball in the environment. These robots have localized themselves, and they are watching the ball, but they cannot observe each other owing to their limited viewing angles (Fig. 1). If we ignore the localization errors and put observations onto a map, the ball positions from two robots may not coincide with each other as shown in Fig. 2(a).

If we use the weighted average of the ball location $\hat{\boldsymbol{x}}_{b a l l}$,

$$
\hat{\boldsymbol{x}}_{\text {ball }}=\frac{{ }^{B} \sigma^{B} \boldsymbol{x}_{\text {ball }}+{ }^{A} \sigma_{\text {ball }}{ }^{A} \boldsymbol{x}_{\text {ball }}}{{ }^{A} \sigma_{\text {ball }}+{ }^{B} \sigma_{\text {ball }}},
$$

where ${ }^{i} \boldsymbol{x}_{j}$ and ${ }^{i} \sigma_{j}$ denote a position and its deviation of the object $j$ estimated by the robot $i$ assuming a Gaussian distributions of the position error. ' indicates that it is the result of information sharing. Then, we have a map shown in Fig. 2(b). The ball position is unified in this map. However, this may not be the true position. Further, when robot A has an almost correct estimation but robot B does not, robot A's estimation becomes worse because of the information sharing. Also there are cases in which the relative position of the robot itself is more important than its absolute position in the world coordinate system. Moreover, it becomes more complicated when the robots can observe each other. If we can assume that the simultaneous observations from several robots are available, then we could use a kind of geometrical constraint, such as the one used by $[4,5]$. For moving robots that have cameras with limited viewing angles, this assumption does not hold.

We propose that each robot believes its observations, constructs its subjective map, and determines its action based on it. For example, robot A believes its observation about the ball and calculates the position of robot $\mathrm{B}$ from the relative position between the ball and robot $\mathrm{B}$ as,

$$
\begin{aligned}
{ }^{A} \hat{\boldsymbol{x}}_{A} & ={ }^{A} \boldsymbol{x}_{A}, \\
{ }^{A} \hat{\boldsymbol{x}_{\text {ball }}} & ={ }^{A} \boldsymbol{x}_{\text {ball }}, \\
{ }^{A} \hat{\boldsymbol{x}}_{B} & ={ }^{B} \boldsymbol{x}_{B}+\left({ }^{A} \boldsymbol{x}_{\text {ball }}-{ }^{B} \boldsymbol{x}_{\text {ball }}\right) .
\end{aligned}
$$




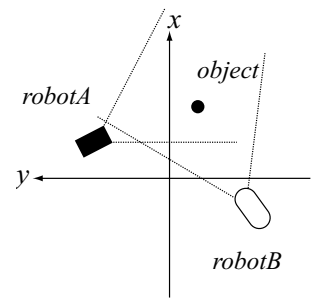

Figure 1: There are two robots watching the ball.

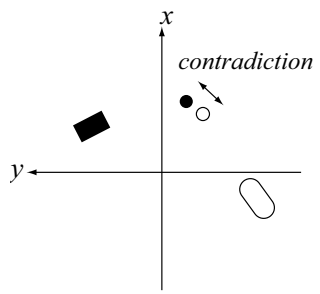

(a) no localization

error assumption

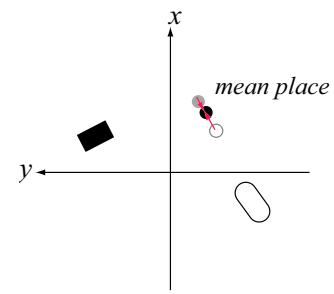

(b) averaged map

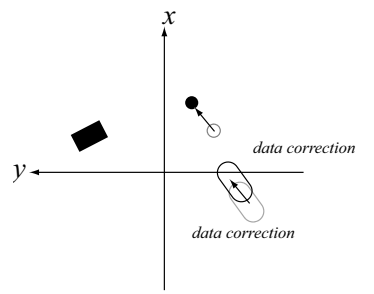

(c) subjective map for robot A

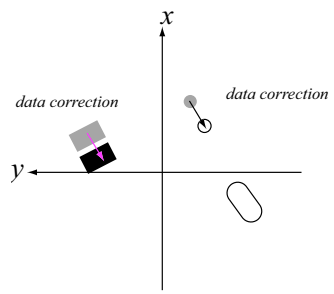

(d) subjective map for robot B

Figure 2: Constructed maps based on the assumption of no localization errors, with averaged locations, and subjective maps of robots $\mathrm{A}$ and $\mathrm{B}$

Figs. 2 (c) and (d) show the subjective maps of robots A and B. With these subjective maps, although reduction of the localization error is not achieved, the localization error of robot B does not affect robot A, and robot A can use the information from robot B. The subjective map method is expected to work for tasks in which relative positions are more important than absolute positions and in which localization errors sometimes become large, and may not satisfy geometrical constraints.

\section{POTENTIAL FIELD for DECISION MAKING}

We define a potential field based on a subjective map of a robot for the experiment we show in the next section. Each robot calculates the field from the map and decides its action based on the field. A robot has four actions: move forward, turn left, turn right, and shoot the ball. The robot selects an action so that it climbs the potential field and its orientation directs to the peak of the potential or to the ball if it is on the peak. Its head is rotated to track the ball if it sees it, or to search for the ball. If the ball is near to the robot, it shoots the ball at the opponent goal.

The potential field $V(x, y)$ of robot $i$ consists of three potentials. One is $V_{T}$, which is a function of the position of a teammate $j,{ }^{i} \boldsymbol{x}_{T j}$. The second is $V_{O}$, which is a function of the position of an opponent $k,{ }^{i} \boldsymbol{x}_{O k}$. The last one is $V_{B}$, which is a function of the ball position ${ }^{i} \boldsymbol{x}_{\text {ball }}$. All the positions are derived from its subjective map. The robot uses its observation for ${ }^{i} \boldsymbol{x}_{O k}$ and ${ }^{i} \boldsymbol{x}_{\text {ball }}$ if it sees them. Otherwise it 


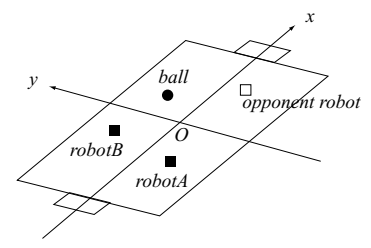

Figure 3: True object locations

uses the position from its teammate for the ball or the opponent position which is subjectively shifted from the information by the teammate based on the ball position as in equation (4). In the following, we give example potentials based on the setup shown in Fig. 3.

Potentials by a teammate $V_{T}$ and an opponent $V_{O}$ are calculated by

$$
\begin{aligned}
V_{T}(\boldsymbol{x}) & =-\sum_{j(j \neq i)} f\left(\boldsymbol{x},{ }^{i} \boldsymbol{x}_{T j}, \boldsymbol{\sigma}_{T}\right), \\
V_{O}(\boldsymbol{x}) & =-\sum_{k} f\left(\boldsymbol{x},{ }^{i} \boldsymbol{x}_{O k}, \boldsymbol{\sigma}_{O}\right), \\
f(\boldsymbol{x}, \overline{\boldsymbol{x}}, \boldsymbol{\sigma}) & =\frac{1}{2 \pi \sigma_{x} \sigma_{y}} e^{-\frac{1}{2} \cdot\left(\left(\frac{x-\bar{x}}{\sigma_{x}}\right)^{2}+\left(\frac{y-\bar{y}}{\sigma y}\right)^{2}\right)} .
\end{aligned}
$$

These potentials are for avoiding robots in the field. Figs. 4 (a), (b), (e) and (f) show the $V_{T}$ and $V_{O}$ of robots A and B in the example setup respectively.

The potential from the ball is defined so that the robot that is closest to the ball can reach it, and the other robots can go to positions where they can back up the shoot. The potential function of robot $i$ is switched depending on whether $i$ is closest to the ball or not,

$$
V_{B}(\boldsymbol{x})= \begin{cases}f\left(\boldsymbol{x},{ }^{i} \boldsymbol{x}_{\text {ball }}, \boldsymbol{\sigma}_{\text {ball }}\right) & (\text { i is closest }), \\ f\left(\boldsymbol{x},{ }^{i} \boldsymbol{x}_{\text {sup }}, \boldsymbol{\sigma}_{\text {sup }}\right) & \text { (otherwise }),\end{cases}
$$

where ${ }^{i} \boldsymbol{x}_{\text {sup }}$ is the support position. It is defined as ${ }^{i} \boldsymbol{x}_{\text {sup }}=\left({ }^{i} \boldsymbol{x}_{\text {ball }}+\boldsymbol{x}_{G}\right) / 2$, where $\boldsymbol{x}_{G}$ is the position of the target goal. The example potentials of robots A and B are shown in Figs. 4 (c) and (g). Final potential fields are shown in Figs. 4 (d) and (h).

\section{EXPERIMETNS}

We used the competition field and the robots for RoboCup Sony four-legged robot league 2002 (Fig. 5) for the experimental setup. In the environment there are six landmark poles, two goals that can be used for self-localization, and one ball. The task is to shoot the ball into the goal. Owing to inadequate lighting conditions and the low resolution of the camera, it was not possible for the robots to stably detect each other in their local vision systems. The self-localization program is based on Carnegie Melon University's CM-Pack'01 [8]. Their multi-hypothesis approach tracks its position by means of a Kalman-filter, starting from different initial positions in the field. To verify the method, we put a color 


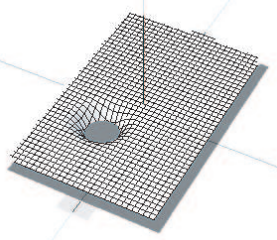

(a) $V_{T}$ of robot $\mathrm{A}$

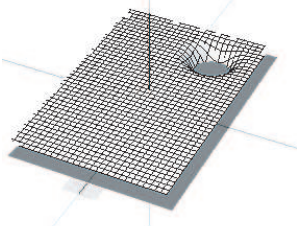

(b) $V_{O}$ of robot $\mathrm{A}$

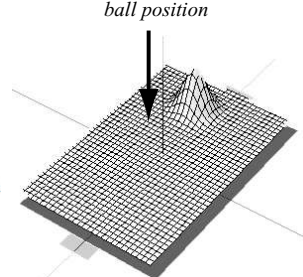

(c) $V_{B}$ of robot A, highest at support position

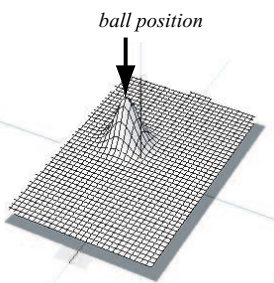

(g) $V_{B}$ of robot B, highest at the ball

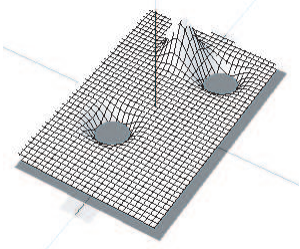

(d) final potential field of robot $\mathrm{A}$

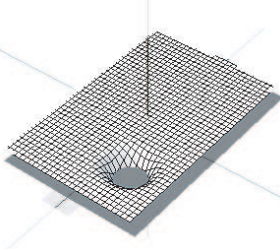

(e) $V_{T}$ of robotB

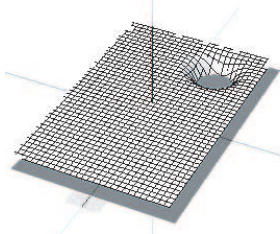

(f) $V_{O}$ of robot B

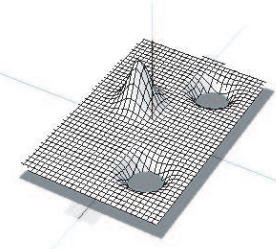

(h) final potential field of robot B

Figure 4: Potential fields of robots.

marker on the back of each robot and used an overhead camera to measure the position of the robots and the ball.

We used two robots A and B and compared the decisions of robot A with the following methods,

[I] robots do not share the information;

[II] robot B sends the information to robot A, and robot A uses the shared map constructed by taking the average of the ball position; and

[III] robot B sends the information to robot A, and robot A uses its subjective map (proposed).

We counted the number of times that the decision of robot A was identical to the decision when robot A is given the location of itself, robot B, and the ball by an overhead camera (OC) and compared the rate,

$$
r=\frac{(\# \text { of times that decision was identical to } O C)}{(\# \text { of total decision times in the trial) }}
$$

Each trial ended after two minutes had elapsed or after the ball had been kicked into the goal.

Figs. 6 (a) and (b) show two initial placements in the experiment. In these initial placements, both robots observe the ball. We have experimented with three methods under two conditions, 1) robot B localizes itself by local vision (normal), and 2) robot B localizes itself but has large errors. Under "normal" error condition, it has $0[\mathrm{~mm}]$ to $80[\mathrm{~mm}]$ offset errors in localization dependent on the 


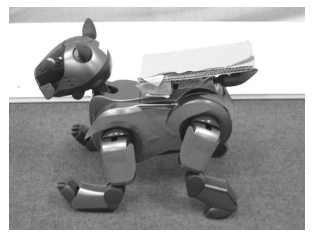

(a) robot

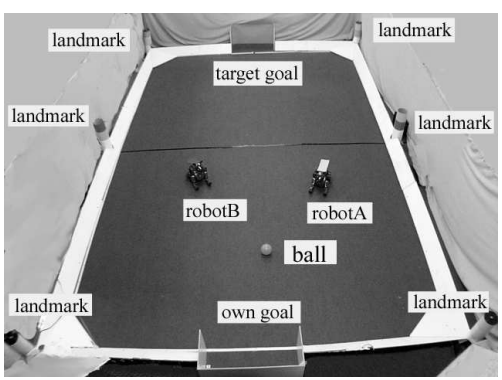

(b) field

Figure 5: The robot and the field for the RoboCup 2002 Sony four-legged robot league.

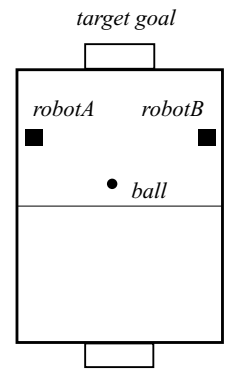

(a) case 1

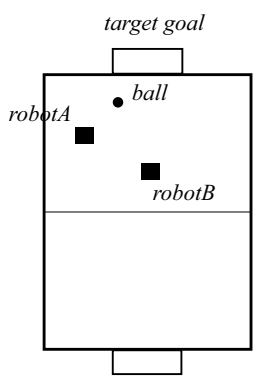

(b) case 2

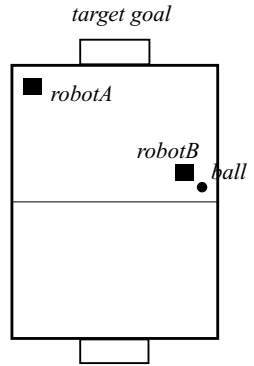

(c) case 3

Figure 6: The initial conditions of experiments.

position and standard deviation is about $20[\mathrm{~mm}]$. Under "large" error condition, robot B's location is inverted against the center of the field, and the 100 degrees offset is added to the orientation. The error in relative distance to the ball varies with its distance and the maximum deviation is about $50[\mathrm{~mm}]$ under both error conditions. We had ten trials for each initial placement in these six conditions. Table 1 shows the rate at which the robot A's decision was identical to the decision with the overhead camera. We can see that with the averaged shared map, when large errors are introduced, the rate is worse than the rate without information sharing. However, with the subjective map, in all conditions, the rate is better than the rate without information sharing and the rate with an averaged shared map. This indicates the validity of the subjective map approach.

In case 1, without information sharing, the robots bumped into each other because they did not know each other's positions, and they were initially placed at nearly the same distance from the ball. With information sharing under normal localization errors, they showed cooperative behaviors. Fig. 7 shows such behaviors.

In case 2, the rate of averaged map showed little difference to that of no information sharing. While under conditions that robot B had large localization errors, the result for the averaged map and 
Table 1: The rate of times that robot A's decision was identical to the decision using an overhead camera

\begin{tabular}{|l|l||c|c|}
\hline \multicolumn{2}{|c|}{ error and method } & \multicolumn{2}{c|}{ the rate } \\
\cline { 3 - 4 } & mean & variance \\
\hline \hline \multirow{3}{*}{$1)$ normal } & {$[\mathrm{I}]$ no sharing } & 0.48 & 0.02 \\
\cline { 2 - 4 } & {$[\mathrm{II}]$ averaged } & 0.64 & 0.01 \\
\cline { 2 - 4 } & {$[\mathrm{III}]$ subjective } & 0.69 & 0.01 \\
\hline \multirow{3}{*}{$2)$ large } & {$[\mathrm{I}]$ no sharing } & 0.48 & 0.02 \\
\cline { 2 - 4 } & {$[\mathrm{II}]$ averaged } & 0.39 & 0.01 \\
\cline { 2 - 4 } & {$[\mathrm{III}]$ subjective } & 0.59 & 0.02 \\
\hline
\end{tabular}

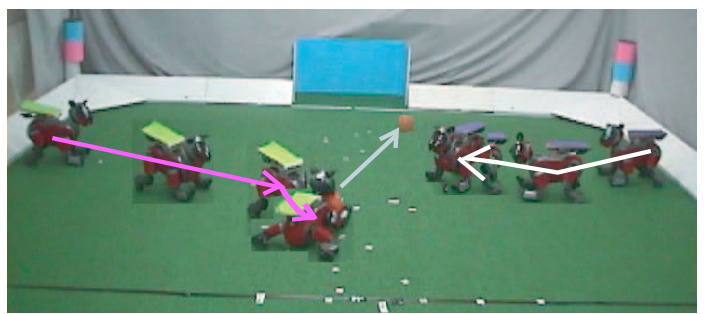

Figure 7: A sequence of the robots' movements using subjective maps in case 1 under normal localization error

subjective map showed a big diffrence. Figs. 8 (a) and (b) show the potential field of robot A and the positions of objects in its averaged map and its subjective map at the initial position. Owing to robot B's large localization errors, robot A also came to have large errors in the averaged map and made incorrect decisions. However, as shown in Fig. 8(b), in the subjective map, robot B's error did not affect robot $\mathrm{A}$, and the rate increased. Fig. 10 shows the robots' movements based on the subjective map. We can see that robot A appropriately moved to the supporting position.

Fig. 6(c) shows the initial placement of case 3. Robot B localizes itself by local vision. In this case, robot A cannot observe the ball but robot B observes it. Then, robot A uses the robot B's position that robot $\mathrm{B}$ tells even when it adopts a subjective map. And robot A has the same map with methods [II] and [III].

Figs. 11 (a) and (b) show the potential field and the positions of objects with a non-shared map and the subjective map in case 3 . Without information sharing, robot A did not know the location of the ball (Fig. 11(a)) nor could it move to the support position. With the subjective map, robot A moved to the support position as expected. A sequence of movements is shown in Fig. 12. In the whole sequence of this figure, robot B aligns itself to the ball. 


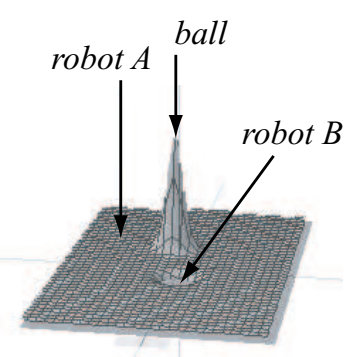

(a) averaged map

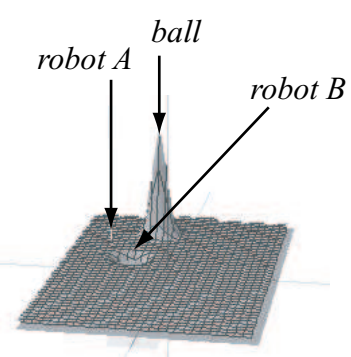

(b) subjective map

Figure 8: Robot A's potential field and the positions of objects in its map at the initial condition in case 2 with robot B's large errors.

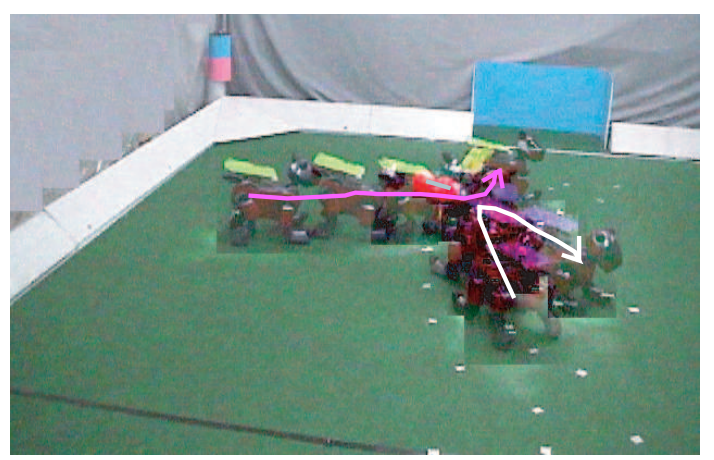

Figure 9: A sequence of the robots' movements using an averaged map in case 2 with large error for robot B.

\section{DISCUSSIONS and CONCLUSIONS}

We have proposed a subjective map in a multi-robot system in a dynamically changing environment. Although the proposed subjective map is rather simple, our experiments showed that even in situations with large errors in the position and the orientation. Under such situations, although a shared averaged map method lost the benefit of information sharing, the proposed subjective map worked effectively.

The idea of a subjective map is independent of global positioning. We have used global positioning in communication since it provided the simplest common coordinate system. When robots can observe several objects at the same time (e.g., each other and a landmark), the relative coodinate on some object is a good candidate for a reference coordinate system, and we need not use a global positioning system. If the quality of information is poor, a qualitative positioning system may work better than a quantitative positioning system.

Future work would involve the investigation of using a subjective map for other kinds of tasks, experiments in more complex multi-robot systems, comparison to other information sharing methods, and the weighting of information based on its reliability measured through communication. 


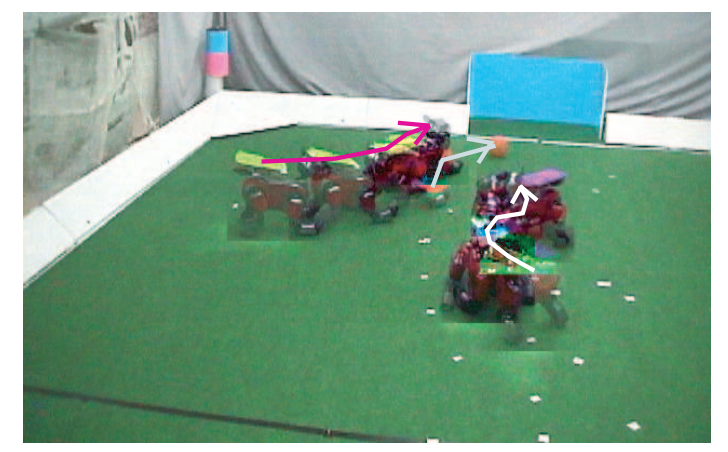

Figure 10: A sequence of the robots' movements using a subjective map in case 2 with large error for robot B.

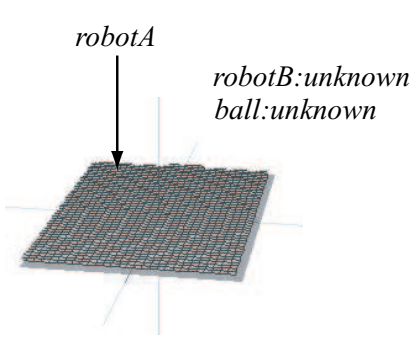

(a) no information share

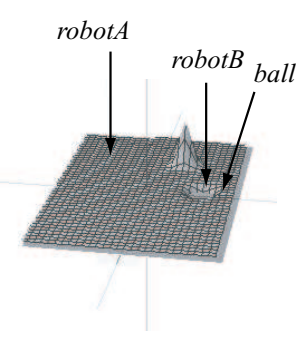

(b) subjective map

Figure 11: Robot A's potential field and positions of objects in its map at the initial condition in case 3.

\section{ACKKNOWLEGEMENT}

We used the codes shared among the teams involved in RoboCup Sony four-legged robot league. We thank Carnegie Melon University for their self-localization codes, the United Team of Kyushu for their motion and walking codes, and the University of New South Wales for their walking codes.

This research was supported by the Japan Science and Technology Corporation's Core Research for the Evolutional Science and Technology Program (CREST) titled Robot Brain Project.

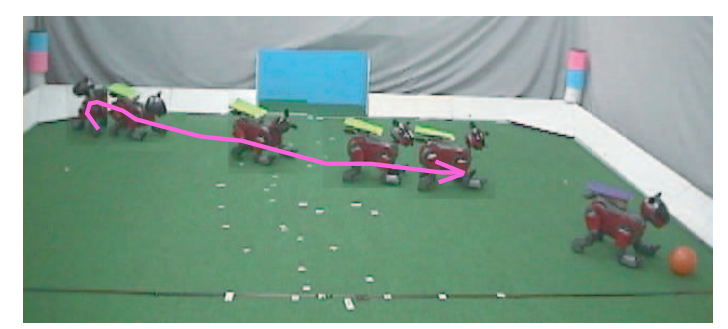

Figure 12: A sequence of the robots' movements with a subjective map in case 3 . 


\section{REFERENCES}

[1] Manuela Veloso, William Uther, Masahiro Fujita, Minoru Asada, and Hiroaki Kitano. Playing soccer with legged robots. In Proceedings of the 1998 IEEE/RSJ International Conference on Intelligent Robots and Systems, volume 1, pages 437-442, 1998.

[2] Ryo Kurazume and Shigeo Hirose. An experimental study of a cooperative positioning system. Autonomous Robots, 8(1):43-52, 2000.

[3] Yasushi Yagi, Shinichi Izuhara, and Masahiko Yachida. The integration of an environmental map observed by multiple mobile robots with omnidirectional image sensor copis. In Proceedings of the 1996 IEEE/RSJ International Conference on Intelligent Robots and Systems, volume 2, pages 640-647, 1996.

[4] K. Kato, H. Ishiguro, and M. Barth. Identifying and localizing robots in a multi-robot system environment. In Proceedings of the 1999 IEEE/RSJ International Conference on Intelligent Robots and Systems, pages 966-971, 1999.

[5] T. Nakamura, A. Ebina, M. Imai, T. Ogasawara, and H. Ishiguro. Real-time estimating spatial configuration between multiple robots by triangle and enumeration constraints. In Proceedings of the 2000 IEEE/RSJ International Conference on Intelligent Robots and Systems, pages 2048-2054, 2000.

[6] Dieter Fox, Wolfram Burgard, Hannes Kruppa, and Sebastian Thrun. A probablistic approach to collaborative multi-robot localization. Autonomous Robots, 3(8), 2000.

[7] Scott Lenser and Manuela Veloso. Sensor resetting localization for poorly modeled mobile robots. In Proceedings of ICRA-2000, 2000.

[8] William Uther, Scott Lenser, James Bruce, Martin Hock, and Manuela Veloso. CM-Pack'01: Fast legged robot waking, robust localization, and team behaviors. In Andreas Birk, Silvia Coradeschi, and Satoshi Tadokoro, editors, RoboCup 2001: Robot Soccer World Cup V, pages 693-696. Springer, Lecture Note in Artificail Intelligence (2377), 2002. 


\section{List of Figures}

1 There are two robots watching the ball. . . . . . . . . . . . . . . . . . 4

2 Constructed maps based on the assumption of no localization errors, with averaged locations, and subjective maps of robots $\mathrm{A}$ and $\mathrm{B} \ldots \ldots \ldots \ldots$. . . . . . . . . . 4

3 True object locations . . . . . . . . . . . . . . . . . . . . . 5

4 Potential fields of robots. . . . . . . . . . . . . . . . . . . . . 6

5 The robot and the field for the RoboCup 2002 Sony four-legged robot league. . . . . . . 7

6 The initial conditions of experiments. . . . . . . . . . . . . . . 7

7 A sequence of the robots' movements using subjective maps in case 1 under normal

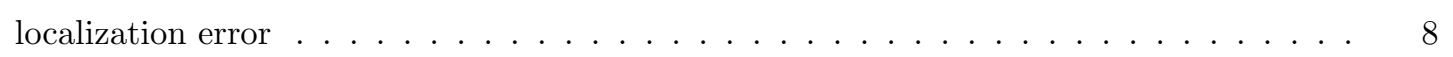

8 Robot A's potential field and the positions of objects in its map at the initial condition in case 2 with robot B's large errors. . . . . . . . . . . . . . . . . . 9

9 A sequence of the robots' movements using an averaged map in case 2 with large error

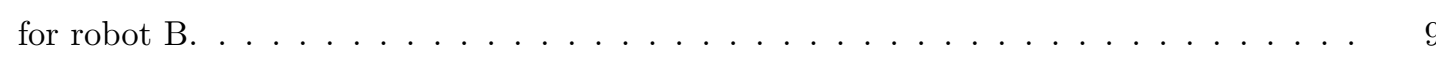

10 A sequence of the robots' movements using a subjective map in case 2 with large error for robot B. . . . . . . . . . . . . . . . . . . . 10

11 Robot A's potential field and positions of objects in its map at the initial condition in case $3 . \ldots \ldots \ldots \ldots \ldots \ldots \ldots \ldots$

12 A sequence of the robots' movements with a subjective map in case 3. . . . . . . . . . . 10 


\section{List of Tables}

1 The rate of times that robot A's decision was identical to the decision using an overhead



